\title{
Surgical resection for liver cancer during the COVID-19 outbreak
}

\author{
Haitham Triki ${ }^{1} \cdot$ Heithem Jeddou ${ }^{1} \cdot$ Karim Boudjema $^{1}$ \\ Received: 30 April 2020 / Accepted: 11 May 2020 / Published online: 20 May 2020 \\ (C) Italian Society of Surgery (SIC) 2020
}

\section{Manuscript}

Since the beginning of 2019 novel coronavirus disease (COVID-19) epidemic in France, caused by severe acute respiratory syndrome coronavirus 2 (SARS-COV-2), hospital capacity, in the most affected regions, has rapidly exceeded. In our department, approximately 250 hepatectomies are performed each year. Decisions to postpone scheduled surgery for liver cancer patients must be made.

Since available data are scarce, many questions have arisen vis-à-vis the management of these patients in the context of this pandemic. Zhang $\mathrm{C}$ et al. reported a detailed discussion about liver injury in COVID-19 [1]. In fact, $14-53 \%$ of COVID-19 patients present abnormal levels of aminotransferases. The degree of liver damage depends on the severity of COVID-19. It seems that cytokine storm syndrome, ischemic hypoxia related to pneumonia and pharmacologic liver injury are the main causes of elevated liver enzymes. However, it has been demonstrated that SARSCOV-2 binds angiotensin converting enzyme 2 (ACE2) receptor to infect the cells. In addition to lung tissue, the ACE2 receptor has also been found in cholangiocytes while the hepatocytes expressed it weakly [1]. Interestingly, it has been shown that ACE2 expression is significantly increased with chronic liver injury and cirrhosis, not only in cholangiocytes but also in hepatocytes [2]. It has been demonstrated in a mouse model after hemi-hepatectomy that ACE2 expression is upregulated during hepatocyte proliferation. Thus, nascent hepatocytes expressing ACE2 receptor could be susceptible to SARS-COV-2 infection [3].

Although it is clear that COVID-19 may cause various degrees of liver damage, many questions remain to be answered about surgery for liver cancer during this pandemic.

Karim Boudjema

karim.boudjema@chu-rennes.fr

1 Department of Hepatobiliary and Digestive Surgery, Pontchaillou Hospital, Rennes 1 University, 35000 Rennes, France

\section{Does COVID-19 increase the risk of morbidity and mortality after liver resection?}

After hepatectomy, the most life-threatening complication is post-hepatectomy liver failure (PHLF). It is known that sepsis is a major cause of PHLF. It affects Kupffer-cell function and increases the concentration of liver cytokines. leukocyte-Kupffer cell interaction leads to the release of TNF $\alpha$ and IL6 [4]. In a similar way, once the cascade of inflammatory reactions is triggered by SARS-COV-2 or in case of related bacterial infection, COVID-19 could be responsible for the development of PHLF. In addition, it has been found that lung injury (a common complication of COVID-19) is an independent risk factor for major morbidity and mortality after hepatectomy [5].

Current antiviral or antimalarial drugs used for the treatment of COVID-19, as well as antipyretic drugs that contain acetaminophen, are metabolized in the liver and could deteriorate PHLF.

\section{Does liver resection further deteriorate the prognosis and complicate management of patients with COVID-19?}

After partial hepatectomy in rats, secretion of TNF- $\alpha$ and IL-6 from the remnant liver increases [6]. High concentrations of circulating TNF- $\alpha$ and IL- 6 were also found in post-hepatectomy infections of hematoma or bilioma [7]. Therefore, hepatectomy and post-hepatectomy infectious complications could further exacerbate the severe COVID19 inflammatory response.

Pulmonary complications are common after hepatectomy. Large subcostal incisions, prolonged surgery, intraoperative blood transfusion, vascular clamping and subsequent ischemia-reperfusion injury are risk factors for pulmonary injury $[8,9]$. Moreover, the use of vasopressors, commonly used in case of Pringle maneuver and inferior vena cava clamping during hepatectomy, is a risk factor for pulmonary 
injury [8]. Hence, liver resections, especially major or complex hepatectomies, in COVID-19 patients could further deteriorate lung damage and complicate its management.

\section{In conclusion, should we keep operating on scheduled liver cancer patients?}

There is some evidence to suggest that liver resection in patients with COVID-19, could aggravate the severity of COVID-19 and complicate its management. Conversely,

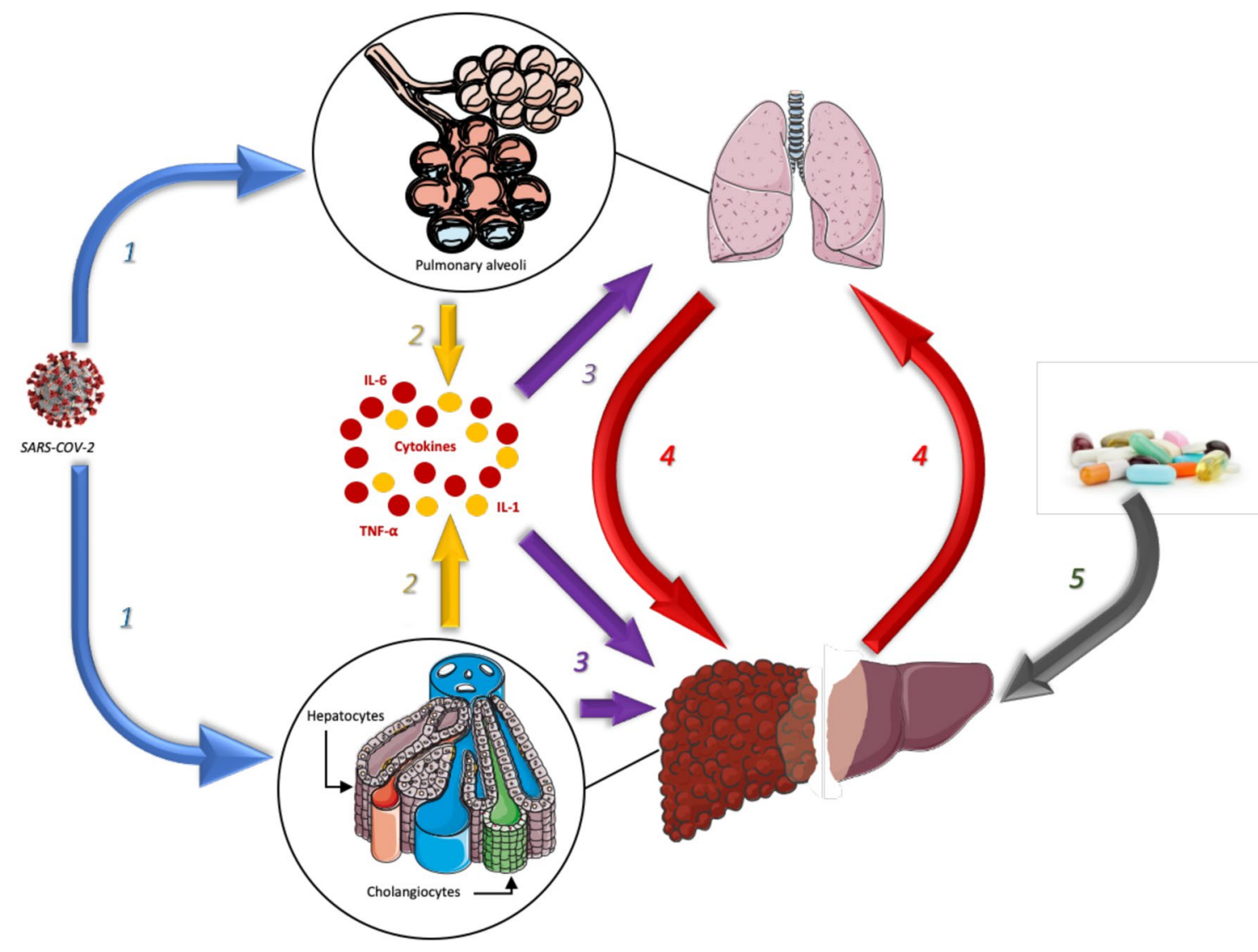

1. SARS-COV-2 binds ACE2 receptor to infect cells. Cholangiocytes express ACE2 receptor. In injured liver (cirrhosis, after hepatectomy), nascent hepatocytes express ACE2 receptor.

\section{Activation of innate and adaptive immune system. Release of cytokine storm.}

3. Inflammatory response induces pulmonary and liver injuries. Liver cells infection by SARS-COV-2 could induce liver injury.

4. Hepatectomy, post-operative infections, pulmonary complications and PHLF may aggravate the severity of pulmonary injury in COVID 19 patient. Ischemic hypoxia caused by pulmonary injury in COVID 19 patient may induce liver injury and PHLF.

5. Drugs may induce liver injury and PHLF. PHLF increases drugs toxicity.

Fig. 1 Liver resection and COVID 19 possible mechanisms for mutual aggravation: COVID-19 2019 novel coronavirus disease, $S A R S-C O V-2$ severe acute respiratory syndrome coronavirus $2, A C E 2$
Angiotensin-Converting Enzyme 2, PHLF post hepatectomy liver failure, $I L-1$ Interleukin-1, TNF- $\alpha$ Tumor necrosis factor- $\alpha$, IL-6: Interleukin-6 
COVID-19 may increase the risk of morbidity and mortality after hepatectomy (Fig. 1).

Due to the risk of contamination by SARS-COV-2 before, during or after hospitalization, we believe that liver surgery for cancer should be postponed for patients at high risk of postoperative complications and severe COVID-19, including elderly patient with comorbidities and chronic liver disease. For these high-risk patients, an alternative treatment, when available, should be undertaken. On the other hand, minor and simple resections ( $\leq 3$ segments) i.e. left lobe laparoscopic resection for a small cholangiocarcinoma or colorectal liver metastases developed on a normal liver in a relatively young patient without comorbidities could be authorized during this outbreak.

Finally, it is urgent to understand the impact of COVID19 in liver cancer patients who undergo surgery. Therapeutic strategies must be adapted to this pandemic, balancing the benefit of liver cancer resection against the risk incurred by the patient during the COVID-19 outbreak.

Author contributions HT, HJ and KB have designed the study and wrote the manuscript.

Funding The authors received no financial support for the research, authorship, and/or publication of this article.

\section{Compliance with ethical standards}

Conflict of interest The authors of this manuscript have no conflicts of interest to disclose as described by the Updates in Surgery.

Research involving human participants and/or animals This article does not contain any research with human and/or animals performed by any of the authors.

Informed consent For this letter to the editor, no informed consent was needed. The authors did not perform any procedures on patients in this manuscript.

\section{References}

1. Zhang C, Shi L, Wang FS (2020) Liver injury in COVID-19: management and challenges. Lancet Gastroenterol Hepatol 5:428-430

2. Paizis G, Tikellis C, Cooper ME, Schembri JM, Lew RA, Smith AI et al (2005) Chronic liver injury in rat and man upregulates the novel enzyme angiotensin converting enzyme II. Gut 54:1790-1796

3. Guan GW, Gao L, Wang JW, Wen XJ, Mao TH, Peng SW et al (2020) Exploring the mechanism of liver enzyme abnormalities in patients with novel coronavirus-infected pneumonia. Zhonghua Gan Zang Bing Za Zhi 28:E002. https://doi.org/10.3760/cma.j.i ssn.1007-3418.2020.02.002

4. Garcea G, Maddern GJ (2009) Liver failure after major hepatic resection. J Hepatobiliary Pancreat Surg 16:145-155

5. Tzeng CW, Cooper AB, Vauthey JN, Curley SA, Aloia TA (2014) Predictors of morbidity and mortality after hepatectomy in elderly patients: analysis of 7621 NSQIP patients. HPB (Oxford) 16:459-468

6. Iwai M, Cui TX, Kitamura H, Saito M, Shimazu T (2001) Increased secretion of tumour necrosis factor and interleukin 6 from isolated, perfused liver of rats after partial hepatectomy. Cytokine 13:60-64

7. Calandra T, Gerain J, Heumann D, Baumgartner JD, Glauser MP (1991) High circulating levels of interleukin-6 in patients with septic shock: evolution during sepsis, prognostic value, and interplay with other cytokines. The Swiss-Dutch J5 Immunoglobulin Study Group. Am J Med 91:23-29

8. Lepere V, Vanier A, Loncar Y, Lemoine L, Vaillant JC, Monsel A et al (2017) Risk factors for pulmonary complications after hepatic resection: role of intraoperative hemodynamic instability and hepatic ischemia. BMC Anesthesiol 17:84

9. Nobili C, Marzano E, Oussoultzoglou E, Rosso E, Addeo P, Bachellier $P$ et al (2012) Multivariate analysis of risk factors for pulmonary complications after hepatic resection. Ann Surg 255:540-550

Publisher's Note Springer Nature remains neutral with regard to jurisdictional claims in published maps and institutional affiliations. 